\title{
Turbulent Diamagnetism in Flowing Liquid Sodium
}

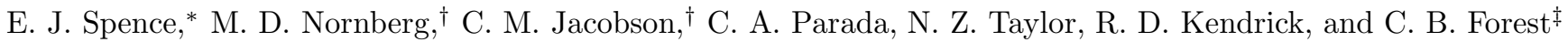 \\ Department of Physics, University of Wisconsin-Madison, \\ 1150 University Avenue, Madison, Wisconsin 53706
}

(Dated: August 16, 2018)

\begin{abstract}
The nature of Ohm's law is examined in a turbulent flow of liquid sodium. A magnetic field is applied to the flowing sodium, and the resulting magnetic field is measured. The mean velocity field of the sodium is also measured in an identical-scale water model of the experiment. These two fields are used to determine the terms in Ohm's law, indicating the presence of currents driven by a turbulent electromotive force. These currents result in a diamagnetic effect, generating magnetic field in opposition to the dominant fields of the experiment. The magnitude of the fluctuation-driven magnetic field is comparable to that of the field induced by the sodium's mean flow.
\end{abstract}

PACS numbers: 47.65.-d, 47.27.-i,91.25.Cw

Keywords: Magnetohydrodynamics, dynamo, Madison Dynamo Experiment

The magnetic fields of stars and planets are generated by the motion of an electrically conducting plasma or liquid metal in the star or planet's interior [1]. The motion of the fluid across an existing seed magnetic field generates a motional electromotive force (EMF) that drives currents, leading to a self-generated magnetic field. While large scale flow may induce much of the magnetic field, there is another means by which magnetic field may be generated: the interaction of velocity and magnetic field fluctuations [2]. If the magnetic and velocity fields are separated into their mean (taken to be the temporally averaged value) and fluctuating components, $\mathbf{B}=\langle\mathbf{B}\rangle+\tilde{\mathbf{B}}$ and $\mathbf{V}=\langle\mathbf{V}\rangle+\tilde{\mathbf{V}}$, then the mean electric current obeys an Ohm's law of the form

$$
\langle\mathbf{J}\rangle=\sigma(\langle\mathbf{E}\rangle+\langle\mathbf{V}\rangle \times\langle\mathbf{B}\rangle+\langle\tilde{\mathbf{V}} \times \tilde{\mathbf{B}}\rangle),
$$

where $\langle\mathbf{J}\rangle$ is the average current density, $\sigma$ the conductivity of the fluid, and $\langle\mathbf{E}\rangle$ the average electric field. The electric field plays a passive role in the scenario studied here, merely maintaining $\nabla \cdot\langle\mathbf{J}\rangle=0$, and as such will not be considered further. There are two significant source terms in Eq. 1: $\langle\mathbf{V}\rangle \times\langle\mathbf{B}\rangle$ represents the EMF associated with the mean part of the velocity and magnetic fields, while $\langle\tilde{\mathbf{V}} \times \tilde{\mathbf{B}}\rangle$ represents the EMF generated by the fluctuating part of the fields; velocity and magnetic field fluctuations can interact coherently to generate mean currents.

The possibility of such a turbulent EMF has long been recognized [2, 3, 4]. Much of its study has focused on those currents generated by helical velocity field fluctuations [5, 6], and several non-simply-connected liquidmetal experiments have been constructed to mimic such helical flows [7, 8]. However, gradients in the intensity of the fluctuations can also generate currents. These currents effectively expel magnetic flux from regions of high turbulence to low, resulting in a diamagnetic effect [2, 9, 10, 11, 12, 13, 14, 15]. Such flux expulsion may explain [16] the weak magnetic field at the center of the galactic core [17] relative to the core's external flux tubes [18], as well as concentrations of large scale toroidal magnetic field at the base of the stellar convection zone [19, 20, 21]. However, since fluctuation-driven fields cannot yet be isolated in astrophysical or geophysical settings, whether turbulent EMFs play a significant role in the production of such magnetic fields remains an open question.

In this Letter we present the spatial structure of such fluctuation-driven currents, as measured in the Madison Dynamo Experiment. The net result is a strong turbulent diamagnetism, reducing the magnitude of the magnetic field throughout the experiment. A previous paper showed that such currents must exist [22], but gave no information about their structure. Here, direct measurement of the experiment's magnetic and velocity fields is used to explicitly examine the structure of Ohm's law.

The Madison Dynamo Experiment is a one-meterdiameter sphere of flowing liquid sodium [23]. Sodium is chosen as the working fluid for its high electrical conductivity. An axisymmetric mean velocity field is generated within the sodium by a pair of counter-rotating impellers attached to shafts that enter the sphere through each pole (with the shafts defining the axis of symmetry). The flowing sodium is very turbulent, with a kinetic Reynolds number $R e \sim 10^{7}$. The unconstrained geometry of the experiment allows fluctuations in the velocity field to develop up to the scale of the device. The fluctuating velocity field generates a fluctuating magnetic field by advecting the mean magnetic field of the experiment.

The nature of Ohm's law is explored by measuring the magnetic-field-dependent terms in Eq. 1 to determine if the induced magnetic field is due solely to the action of the mean flow. An approximately-uniform magnetic field is applied to the flowing sodium using a pair of external magnetic field coils, and the total magnetic field, applied plus induced, is measured (the applied field is sufficiently weak that the velocity field is unaffected). The collection of magnetic field data has been described previously [22]. Since both the mean velocity and applied 

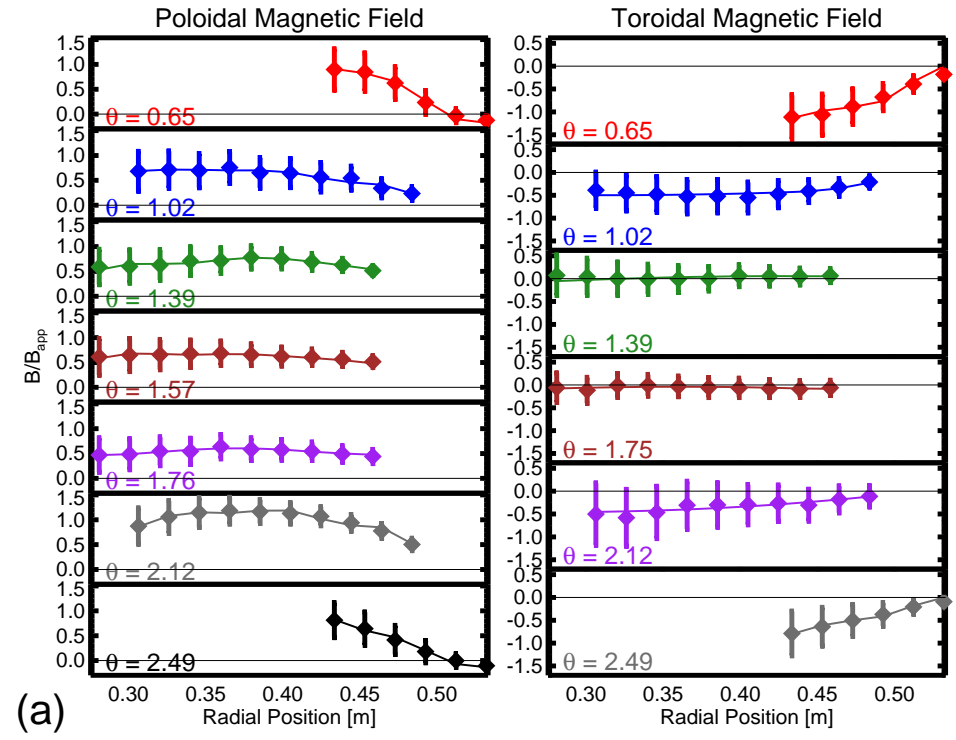

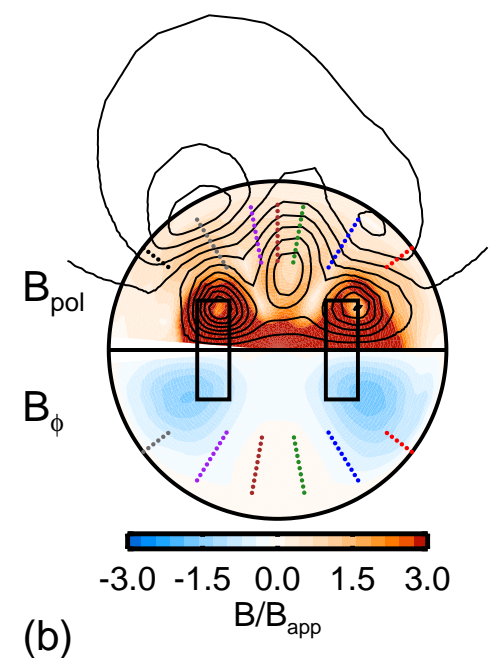

(b)

FIG. 1: Induced magnetic fields measured in the Madison Dynamo Experiment. (a) Mean measured poloidal and toroidal field values, scaled to the magnitude of the applied field (50 Gauss), for an impeller rotation rate of 1000 RPM. The fit (solid lines) represents values predicted by a spherical harmonic expansion fit to the data (diamonds). Error bars represent the RMS fluctuation levels of the signals. (b) The reconstructed field, with the axis of symmetry oriented horizontally. Streamlines of the poloidal field are in the upper hemisphere and the toroidal field strength is in the lower hemisphere. Measurement positions are indicated with dots, and the impeller positions are indicated with rectangles.

magnetic fields are axisymmetric, the mean induced field is also axisymmetric (all data presented in this Letter are axisymmetric). A spherical harmonic expansion of the induced internal magnetic field, in the poloidal and toroidal directions, is fitted to the most probable values of the measured magnetic field. Since the sphere is simply connected, a toroidal magnetic field cannot be applied from outside the sphere; all measured toroidal magnetic field is due to electrical currents flowing within the sodium.

For an impeller rotation rate of 1000 revolutions per minute (RPM), the mean measured induced magnetic field ranges from 1.2 times the magnitude of the applied field in the poloidal direction, to 1.1 times in the toroidal direction [Fig. 1(a)]. The field reconstructed from the fit indicates that the external poloidal magnetic field is dominated by a dipolar component that opposes the externally-applied magnetic field [Fig. 1(b)]. The reconstruction also demonstrates that the toroidal velocity field is effective at generating toroidal magnetic field from the applied poloidal magnetic field.

To distinguish between $\langle\mathbf{B}\rangle_{\langle\mathbf{V}\rangle \times\langle\mathbf{B}\rangle}$, the magnetic field induced by the mean flow $\left(\nabla \times\langle\mathbf{B}\rangle_{\langle\mathbf{V}\rangle \times\langle\mathbf{B}\rangle}=\right.$ $\left.\mu_{0}\langle\mathbf{J}\rangle_{\langle\mathbf{V}\rangle \times\langle\mathbf{B}\rangle} \sim \mu_{0} \sigma\langle\mathbf{V}\rangle \times\langle\mathbf{B}\rangle\right)$, and $\langle\mathbf{B}\rangle_{\langle\tilde{\mathbf{V}} \times \tilde{\mathbf{B}}\rangle}$, the magnetic field due to fluctuations $\left(\nabla \times\langle\mathbf{B}\rangle_{\langle\tilde{\mathbf{V}} \times \tilde{\mathbf{B}}\rangle} \sim\right.$ $\left.\mu_{0} \sigma\langle\tilde{\mathbf{V}} \times \tilde{\mathbf{B}}\rangle\right)$, the mean velocity field of the sodium must be known. To this end an identical-scale water model of the sodium experiment has been constructed [24]. At the correct temperatures water and sodium have the same kinematic viscosity and similar densities. As a result the two fluids are nearly hydrodynamically identical and water can be used to model the flowing sodium. Like the sodium apparatus, the water model is a one-meterdiameter sphere in which impellers generate an axisymmetric mean velocity field. Stainless steel tubes, identical to those containing the internal Hall probes in the sodium experiment 23], enter the flow at the same seven locations as in the sodium experiment. Unlike the sodium apparatus, the water model is outfitted with five windows that allow a Laser Doppler Velocimetry (LDV) system to directly measure the poloidal and toroidal components of the water's velocity field. A spherical harmonic expansion of the velocity field is fitted to the mean values of these measurements to determine the mean velocity field of the flowing sodium. For a impeller rotation rate of 1000 RPM, the measured velocity field data yield a maximum poloidal speed of $2.5 \mathrm{~m} / \mathrm{s}$ and a maximum toroidal speed of $3.9 \mathrm{~m} / \mathrm{s}$ [Fig. 2(a)]. As expected, the flow is counter-rotating in the toroidal direction and the poloidal flow rolls inward at the equator and outward at the poles [Fig. 2(b)].

Once the mean velocity field is known the magnetic field due to the mean flow interacting with the mean magnetic field [Fig. 3(a)] and the magnetic field driven by the fluctuations [Fig. [3(b)] are calculated. Like the fit to the measured magnetic and velocity fields, this calculation is done in a spherical harmonic basis, and involves calculating the electrical potential of the experiment assuming $\nabla \cdot\langle\mathbf{J}\rangle=0$. If no fluctuation-driven currents 

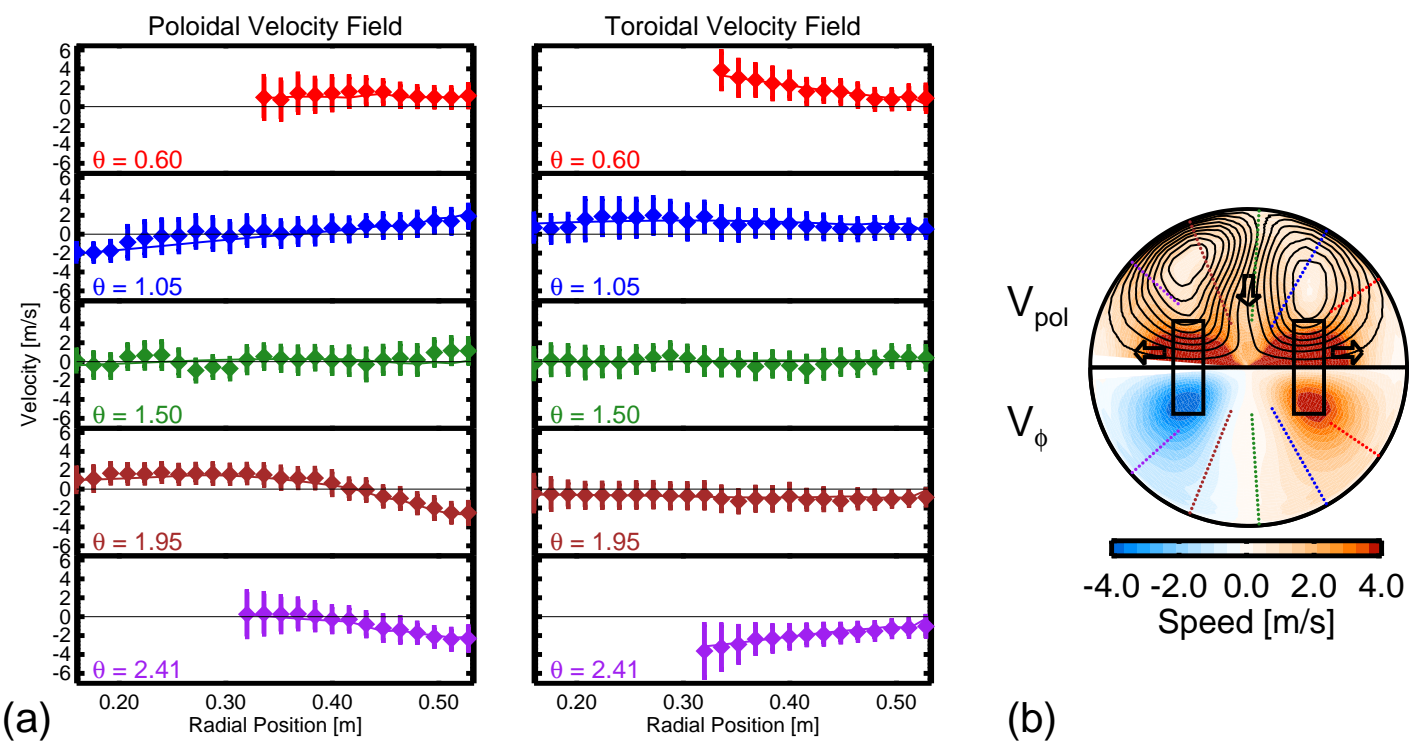

(b)

FIG. 2: Velocity fields measured by Laser Doppler Velocimetry in the water model of the Madison Dynamo Experiment. (a) Mean measured velocity field as a function of radial position, for an impeller rotation rate of 1000 RPM. (b) The reconstructed field. The poloidal flow rolls inward at the equator and outward at the poles. Two toroidal cells rotate in opposing directions in each hemisphere. In both panels the conventions are as in Fig. 1.

were present (i.e. the magnetic field is solely explained by $\left.\langle\mathbf{J}\rangle_{\langle\mathbf{V}\rangle \times\langle\mathbf{B}\rangle} \sim \sigma\langle\mathbf{V}\rangle \times\langle\mathbf{B}\rangle\right)$, then the measured mean magnetic field [Fig. [1(b)] would be the same as the field due to the mean velocity field [Fig. 3(a)]. Inspection of these two figures reveals that this is not the case. The prominent external dipole component of the measured poloidal magnetic field is completely absent from the magnetic field due to the mean velocity field, since the mean axisymmetric velocity field is incapable of producing it 22]. Also, the magnitude of the measured toroidal magnetic field is significantly weaker than the toroidal field due to the mean velocity field interacting with the measured magnetic field. Clearly, the inductive action of the mean flow alone is insufficient to explain the measured fields: fluctuations must be generating significant magnetic field.

The magnetic field generated by the fluctuations has several prominent features [Fig. 3(b)]. First, there is a dipole component that dominates the magnetic field outside the sphere, in the direction opposite the applied field. This is the source of the measured external dipole moment. Second, the toroidal field induced by the fluctuations is in opposition to the field induced by the mean velocity field interacting with the measured magnetic field. Thus, the field induced by the fluctuations is diamagnetic with respect to the dominant poloidal and toroidal fields within the experiment. The effect is important to the overall magnetic field of the experiment, as the fluctuation-driven field is a significant fraction of both the field induced by the mean flow, and the applied field. The strength of the toroidal diamagnetic field near the poles is about $50 \%$ of the magnitude of the field induced by the mean flow, while the fluctuation-driven dipole component is $20 \%$ of the magnitude of the applied field. The fluctuation-driven poloidal field reduces the total poloidal flux through the equatorial plane by $10 \%$.

Characterization of the fluctuations which lead to this turbulent EMF requires direct measurement of the fluctuating components of $\mathbf{V}$ and $\mathbf{B}$. Since such measurements are not currently available, it is not yet possible to determine whether the observed diamagnetism is due to gradients in fluctuation levels, helical turbulence, or some other effect. Since the fluctuation levels of the experiment are greatest near the impellers, and weakest near the sphere's surface, flux expulsion due to gradients in turbulence levels is a natural candidate to explain the observed diamagnetism, though it is not the only possible explanation.

We note that the structure of the diamagnetic field is qualitatively similar to the fluctuation-driven field predicted by recent numerical simulations of the experiment 25]. These simulations predict both the presence of the dipole moment and the overall weakening of the measured toroidal field. However, the magnitude of the fluctuation-driven field presented here is a factor of five larger than that predicted by the simulations. The reason for this discrepancy is not currently known, though it may be related to differences in fluid forcing or the simulation's large value of magnetic Prandtl number.

In summary, mean magnetic and velocity field measurements have been used to determine the structure of fluctuation-driven currents in the Madison Dynamo 

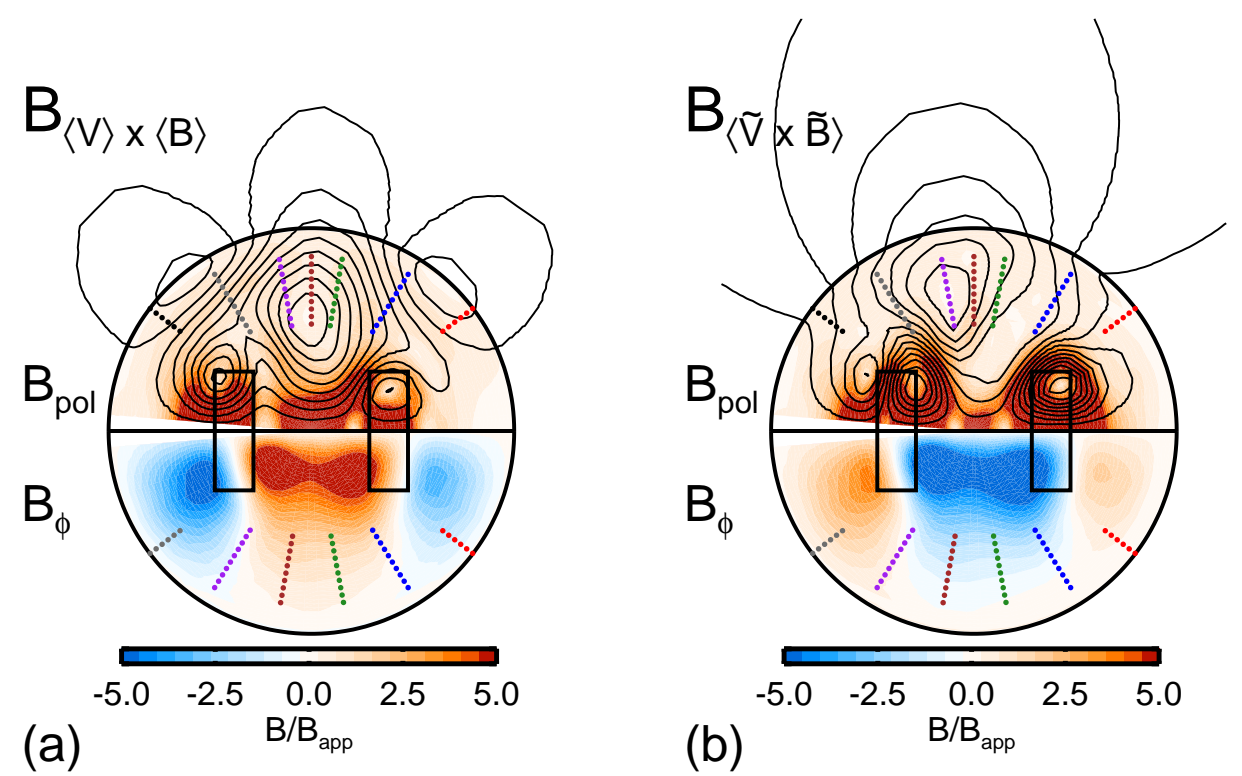

FIG. 3: Magnetic field due to the terms in Ohm's law. (a) The magnetic field, $\langle\mathbf{B}\rangle_{\langle\mathbf{V}\rangle \times\langle\mathbf{B}\rangle}$ calculated from the mean measured velocity field interacting with the measured mean magnetic field (the sum of the induced and the applied fields). Note the lack of induced external dipole moment. (b) The mean magnetic field, $\langle\mathbf{B}\rangle_{\langle\tilde{\mathbf{V}} \times \tilde{\mathbf{B}}\rangle}$, due to the EMF associated with the fluctuations. The poloidal external field displays the measured dipole component, in opposition to the applied field. The toroidal field is in opposition to the field induced by the mean flow of sodium.

Experiment. These currents lead to a magnetic field in opposition to the experiment's dominant magnetic field. This is the first observation of turbulent diamagnetism in a laboratory setting. Analysis of the nature of the fluctuations which leads to this turbulent EMF is ongoing, and future work will focus on further understanding the localized regions of current generation. Given that the observed effect is diamagnetic, it indicates that such fields could be a hindrance to the development of magnetically self-exciting geophysical and astrophysical systems, as well as simply-connected liquid-metal dynamo experiments.

E.J.S. thanks A. Jackson and C. Finlay for reviewing the manuscript. This work is funded by the US Department of Energy, the National Science Foundation, and David and Lucille Packard Foundation.

* Current address: Institut für Geophysik, ETH Zürich, CH-8093 Zürich, Switzerland

† Current address: Princeton Plasma Physics Laboratory, Princeton University, P.O. Box 451, Princeton, New Jersey 08543

¥ Electronic address: cbforest@wisc.edu

[1] G. Rüdiger and R. Hollerbach, The Magnetic Universe: Geophysical and Astrophysical Dynamo Theory (Wiley, New York, 2004), ISBN 3-527-40409-0.

[2] K. Krause and K. H. Rädler, Mean-field Magnetohydrodynamics and Dynamo Theory (Pergammon Press, New York, 1980).
[3] M. Steenbeck and F. Krause, Z. Naturforsch. Teil A 21, 1285 (1966).

[4] H. K. Moffatt, Magnetic field generation in electrically conducting fluids (Cambridge University Press, Cambridge, England, 1978).

[5] E. N. Parker, Astrophys. J. 122, 293 (1955).

[6] F. Krause and M. Steenbeck, Z. Naturforsch. Teil A 22, 671 (1967).

[7] M. Steenbeck, I. M. Kirko, A. Gailitis, A. P. Klyavinya, F. Krause, I. Y. Laumanis, and O. A. Lielausis, Sov. Phys.-Doklady 13, 443 (1968).

[8] R. Stieglitz and U. Müller, Phys. Fluids 13, 561 (2001).

[9] S. I. Vainshtein, Zh. Prikl. Mekh. Tekh. Fiz. 1, 12 (1971).

[10] E. N. Parker, Astrophys. J. 202, 523 (1975).

[11] S. I. Vainshtein and L. L. Kichatinov, Geophys. Astrophys. Fluid Dynam. 24, 273 (1983).

[12] L. Tao, M. R. E. Proctor, and N. O. Weiss, Mon. Not. R. Astron. Soc. 300, 907 (1998).

[13] F. Cattaneo, Astrophys. J. 434, 200 (1994).

[14] S. M. Blanchflower, A. M. Rucklidge, and N. O. Weiss, Mon. Not. R. Astron. Soc. 301, 593 (1998).

[15] M. Meneguzzi, U. Frisch, and A. Pouquet, Phys. Rev. Lett. 47, 1060 (1981).

[16] S. Boldyrev and F. Yusef-Zadeh, Astrophys. J. 637, L101 (2006).

[17] T. N. LaRosa, C. L. Brogan, S. N. Shore, T. J. Lazio, N. E. Kassim, and M. E. Nord, Astrophys. J. 626, L23 (2005).

[18] F. Yusef-Zadeh, Astrophys. J. 598, 325 (2003).

[19] W. Arter, M. R. E. Proctor, and D. J. Galloway, Mon. Not. R. Astron. Soc. 201, 57P (1982).

[20] A. Nordlund, A. Brandenburg, R. L. Jennings, M. Rieutord, J. Roukolainen, R. F. Stein, and I. Tuominen, Astrophys. J. 392, 647 (1992). 
[21] L. Tao, N. O. Weiss, D. P. Brownjohn, and M. R. E. Proctor, Astrophys. J. 496, L39 (1998).

[22] E. J. Spence, M. D. Nornberg, C. M. Jacobson, R. D. Kendrick, and C. B. Forest, Phys. Rev. Lett. 96, 055002 (2006).

[23] M. D. Nornberg, E. J. Spence, R. D. Kendrick, C. M. Jacobson, and C. B. Forest, Phys. Plasmas 13, 055901
(2006).

[24] C. B. Forest, R. A. Bayliss, R. D. Kendrick, M. D. Nornberg, R. O'Connell, and E. J. Spence, Magnetohydrodynamics 38, 107 (2002).

[25] R. A. Bayliss, C. B. Forest, M. D. Nornberg, E. J. Spence, and P. W. Terry (2007), in press, Phys. Rev. E. 